\title{
New Software for Multimodal Image Registration with Application in Cell Tracking Studies
}

Dietz $\mathrm{B}^{1 *}$, Alexander $\mathrm{M}^{2}$, Elhami $\mathrm{E}^{2}$, Xiang $\mathrm{B}^{3}$, Deng $\mathrm{J}^{3}$, Wang $\mathrm{F}^{4}$, Chi $\mathrm{C}^{4}$, Goertzen $\mathrm{AL}^{5}$, Mzengeza $\mathrm{S}^{5}$, Freed $\mathrm{D}^{6}, \mathrm{Arora} \mathrm{RC}^{7}$ and Tian $\mathrm{G}^{3 *}$

${ }^{1}$ Department of Physics and Astronomy, University of Manitoba, Canada

${ }^{2}$ Department of Physics, University of Winnipeg, Canada

${ }^{3}$ National Research Council Canada, Canada

${ }^{4}$ Harbin Medical University, Harbin, People's Republic of China

${ }^{5}$ Department of Radiology, University of Manitoba, Canada

${ }^{6}$ Department of Cardiac Surgery, University of Alberta Hospital, Canada

${ }^{7}$ Cardiac Sciences Program, St. Boniface General Hospital, Canada

\begin{abstract}
Positron Emission Tomography (PET) is an essential tool for in vivo molecular imaging, due to its high sensitivity. Combined with the unparalleled soft tissue contrast of magnetic resonance imaging (MRI), PET-MRI allows for accurate quantification required for cell tracking studies. The purpose of this study is to develop a landmark based image registration program capable of fiducial registration, for application in a stem cell tracking study. PET and MR Images acquired with fiducial markers located in the field of view around the specimen were registered using our landmark based program, written in Matlab. The fiducial markers were also used to test the accuracy of our Matlab program, along with other automated image registration software including: automatic image registration (AIR), FMRIB's Linear Image Registration Tool (FLIRT), and Medical Image Processing, Analysis, and Visualization (MIPAV), all available online free for download. All of the programs were able to successfully register the images; however, our Matlab program provided the best registration accuracy, with a Fiducial Registration Error (FRE) of 1.36 $\mathrm{mm}$. Our Matlab developed program is available free for download at www.sourceforge.net/p/matlabaffinereg.
\end{abstract}

Keywords: Image registration software; Multi-modality PET-MR imaging; Small animal imaging; Cell tracking

\section{Introduction}

Positron emission tomography (PET) is a functional imaging modality that has proven to be an essential tool for in vivo molecular imaging, due to its high sensitivity, allowing for picomolar levels of detection [1]. PET is often used for diagnoses of cancer using the clinically approved radiopharmaceutical $\left[{ }^{18} \mathrm{~F}\right]$ fluoro-2-deoxy-Dglucose (FDG), a glucose analog that remains trapped in the cell during glycolysis [2]. Tumours require high amounts of glucose to proliferate, thereby creating high intensity regions of FDG accumulation that are indicative of cancer [3]. The ability to detect small concentrations of labeled cells makes PET ideal for non--invasive stem cell tracking studies [4]. Unfortunately, PET does not contain enough anatomical information for accurate delineation of tissues. PET data must be registered to anatomical data, such as MRI, to ensure accurate Volumes of Interest (VOI) are used for quantification [5]. Magnetic resonance imaging (MRI) offers high spatial resolution and unparalleled soft tissue contrast [6], which is important for accurate VOI analysis. Furthermore, Superparamagnetic Iron Oxide (SPIO) particles can be used to label cells for MRI cell tracking studies [1].

The combination of PET and MRI offers the high sensitivity detection of PET, along with the high spatial resolution of MRI. Therefore, PET-MRI is ideal for pre-clinical cell tracking studies. Although new scanners allowing for simultaneous PET-MRI image acquisition have been recently introduced (Siemens Biograph mMR), many centers still rely on registration of two separate imaging systems. In order to utilize both functional and anatomical imaging modalities, image registration is required to bring the images into a common reference frame. The purpose of this study is to register PET and MR images of rats that have undergone acute myocardial infarction, followed by an injection of dual-labeled stem cells, and quantify the initial biodistribution of the stem cells in regions such as the heart, lungs, and kidneys.
Intermodal 2D image registration of PET and MR images can be used to accurately locate small concentrations of cells detected using PET within the high spatial resolution of an MR image. There are several transformation methods that can be applied to an image for registration, for example, in 2D: rigid body (3 parameters), affine (6 parameters), or non-linear (no limit to the number of parameters) [7]. Rigid body transformations include translation along the $\mathrm{x}$ - and $y$-axes, as well as rotation. Affine transformations include translation, rotation, skew along the $\mathrm{x}$ - and $\mathrm{y}$-axes, and global scaling. Non-linear transformations have no limit to the number of parameters. For the purpose of our study, we focused only on affine transformations.

This study investigates registration of PET and MRI images using several automatic registration programs available free on the Internet. These include: FMRIB's linear image registration tool (FLIRT) [8], automated image registration (AIR) [9], and medical image processing, analysis and visualization (MIPAV) [10]. The registration accuracy was determined using the fiducial registration error (FRE), which is the distance between fiducial markers after registration has been performed [11]. A perfect registration would ideally yield an FRE of $0 \mathrm{~mm}$. All of the software mentioned above have the ability to achieve accurate image registrations; however, they have their limitations. A major limiting factor required for automatic image registration is that

*Corresponding author: Dietz B, Department of Physics and Astronomy, University of Manitoba, 66 Chancellors Cir, Winnipeg, MB R3T 2N2, Canada, Tel: 1226-984-8715; E-mail: brysondietz@gmail.com

Received August 05, 2014; Accepted October 08, 2014; Published November 10, 2014

Citation: Dietz B, Alexander M, Elhami E, Xiang B, Deng J, et al. (2014) New Software for Multimodal Image Registration with Application in Cell Tracking Studies. J Stem Cell Res Ther 4: 239. doi:10.4172/2157-7633.1000239

Copyright: (c) 2014 Dietz B, et al. This is an open-access article distributed under the terms of the Creative Commons Attribution License, which permits unrestricted use, distribution, and reproduction in any medium, provided the original author and source are credited. 
the images must have a similar structure. In order to overcome such limitations, we have developed a Matlab affine registration (MAR) program that is easy to use, with a simple graphical user interface (GUI), allowing for 2D registration of images containing fiducial markers. For simplicity we have named our program MAR-GUI, which is available free for download at www.sourceforge.net/p/matlabaffinereg.

\section{Methods}

\section{Animal model for heart failure}

The animals used for this study were treated in compliance with the Canadian Council on Animal Care Guide. The experiment protocols were approved by the National Research Council of Canada Animal Care Committee.

The animal model used for this study was a rat acute infarct heart model. The model was created by occlusion of the lower anterior descending coronary artery, inducing myocardial infarction. Duallabeled (FDG-SPIO) Adipose-derived Stem Cells (ASCs) were immediately injected into the animal via one of three injection sites: myocardium, left ventricle, or tail vein [12]. Table 1 contains the experimental details on the amount of cells and activity of the injected stem cells. Several images of rats injected with ASCs were taken at the National Research Council (NRC) located in Winnipeg, MB Canada. Our previous study [13] has shown that the retention of the FDG in the stem cells is acceptable for short term stem cell tracking studies.

\section{Imaging protocols}

For PET and MR imaging, the anesthetized animals were placed on a PET-MR compatible bed in prone position. Several hollow spheres filled with $15 \mathrm{kBq}$ FDG (PET contrast) in $2 \% \mathrm{CuSO}_{4}$ solution (MR contrast) were attached to the bed near the heart region, and serve as fiducial markers for the image registration procedures. The imaging protocols involve an initial scanning with a microPET P4 dedicated animal PET system (Siemens Preclinical Solutions, Knoxville, TN), followed by a 7T MRI scanner. PET images were acquired with an initial 30 minute emission scan of the heart, followed by 10 minute emission scans of the head, heart, and kidneys and bladder. All PET emission images were reconstructed iteratively using an OSEM3D/ MAP algorithm provided by the system manufacturer. After each PET emission scan, a PET transmission scan using a ${ }^{57}$ Co point source was taken. PET transmission images involve rotating a rod source around the PET bed to produce an attenuation map that is used for attenuation correction [14]. The PET-MR compatible bed allows for minimal motion when moving the rat from the PET scanner to the MRI scanner. The bed is designed for a radio frequency $(\mathrm{RF})$ volume coil to slide over it, further minimizing any motion from the rat. MR images were acquired using a FLASH sequence with $1.43 \mathrm{~ms}$ of echo time (TE), $4.6 \mathrm{~ms}$ of repetition time (TR), and $17.5^{\circ}$ of Gaussian excitation pulse. The sequence was ECG-triggered and respiratory gated. The total acquisition time was 10-20 minutes.

\section{Image registration}

Affine transformation: All programs used in this study to register

\begin{tabular}{|c|c|c|}
\hline Injection Site & No. of Stem Cells & Activity \\
\hline Myocardium & $4.6 \pm 2.0$ million & $1.3 \pm 0.9 \mathrm{MBq}$ \\
\hline Left Ventricle & $5.5 \pm 1.5$ million & $1.5 \pm 0.8 \mathrm{MBq}$ \\
\hline Tail Vein & $6.0 \pm 0.8$ million & $3.6 \pm 2.6 \mathrm{MBq}$ \\
\hline
\end{tabular}

Table 1: Number of stem cells measured using the trypan-blue $(0.4 \%)$ exclusion assay and the amount of activity injected as measured using a dose calibrator for three injection methods. Values given in mean \pm standard deviation.
PET and MR images use an affine transformation, which includes translation, rotation, scale and skew parameters.

\section{An affine transformation can be written as $\boldsymbol{y}=\mathbf{A x}+\mathbf{t}$}

Where, $y$ are the transformed coordinates, $x$ are the corresponding initial coordinates, $\boldsymbol{t}$ is the translation vector, and $A$ is the transformation matrix that includes rotation, scale, and skew. In our case, we can imagine $\boldsymbol{x}$ as the initial PET coordinates and $\boldsymbol{y}$ as the transformed PET coordinates. The Matlab code was developed by minimizing the cost function $\mathrm{E}$, with respect to the affine parameters $A, t$, where

$$
E=\sum_{n=1}^{N}\left(\frac{\square \boldsymbol{y}^{(n)}-\left(A \boldsymbol{x}^{(n)}+\boldsymbol{t}\right) \square^{2}}{\sigma_{n}^{2}}+\frac{\square \boldsymbol{x}^{(n)}-\left(B \boldsymbol{y}^{(n)}+\boldsymbol{u}\right) \square^{2}}{\sigma_{n}^{2}}\right)
$$

Here $\|. .$.$\| denotes the Euclidean norm and \mathrm{N}$ denotes the number of fiducials. The terms $B$ and $\boldsymbol{u}$ describe the inverse affine transform from $y$ to $x$. We assume that the variances $\sigma_{n}^{2}$ are the same for each measurement $\boldsymbol{x}^{(\mathrm{n})}, \boldsymbol{y}^{(\mathrm{n})}$.

Fiducial registration: One method for image registration is landmark registration, which makes use of fiducial markers. These are hollow spheres filled with contrast medium visible in both MRI and PET images that act as an independent set of reference points and are used for image registration purposes. The fiducial markers used in our study were filled with a mixture of $18 F$-FDG and copper sulfate $\left(\mathrm{CuSO}_{4}\right)$, contrast agents for imaging with PET and MRI, respectively. Registration of the fiducial markers ensures registration of the entire image, assuming the object remains fixed with respect to the fiducial markers throughout scanning. For the purpose of image registration and validation, images were taken of rats with fiducial markers located around the heart.

The accuracy of our MAR-GUI program was tested against several automatic registration programs as described below. PET and MR images containing three fiducial markers were chosen to test the accuracy of registration for each program. The fiducial markers provide a landmark based method to determine registration accuracy for all registration programs. The fiducial markers were used to perform the registration using our MAR-GUI program. All other automatic registration programs registered the images using various intensity based cost functions, without the fiducials present in the images. The fiducial were removed, as they would generally not be required for intensity based automatic registration programs. The transformations calculated using the various automated registration programs were then applied to the images with fiducials present, whereby landmark accuracy could be determined.

Automatic image registration: Several automatic image registration programs, including FLIRT, AIR, and MIPAV were used to determine the registration accuracy of our images. The images were registered using the various cost functions associated with each of the registration programs. Unless otherwise stated, the default interpolation, optimization and various other settings were used.

The cost functions tested using FLIRT include correlation ratio (CR), normalized cross correlation (NCC), mutual information (MI), and normalized mutual information (NMI). The default interpolation is bilinear and the optimization routine consists of a multi-resolution algorithm. An initial global search at a coarse resolution is followed by several local searches at successively finer resolutions [8].

The cost function tested using AIR is the Woods algorithm, which seeks to maximize the uniformity of the two images. AIR also uses a 
Citation: Dietz B, Alexander M, Elhami E, Xiang B, Deng J, et al. (2014) New Software for Multimodal Image Registration with Application in Cell Tracking Studies. J Stem Cell Res Ther 4: 239. doi:10.4172/2157-7633.1000239

Page 3 of 5

multi-resolution optimization technique; it initially registers every $81^{\text {st }}$ pixel and after convergence, continues with every $27^{\text {th }}$ pixel, followed by every $9^{\text {th }}, 3^{\text {rd }}$, and ultimately every pixel [9]. The default interpolation for AIR is bilinear.

The cost functions investigated using MIPAV include CR, NCC, and NMI. MIPAV uses Powell's method combined with Brent's method for optimization [10]. Powell's method is an optimization algorithm that does not require derivatives, while Brent's method is a root-finding algorithm that is essentially a golden-search optimization method [15]. The default interpolation used in MIPAV is also bilinear.

A drawback to using the custom built bed described above is its presence in the PET transmission images, but not in the MR images. This becomes an issue for registration of PET transmission images to the MR images when using the automatic image registration programs
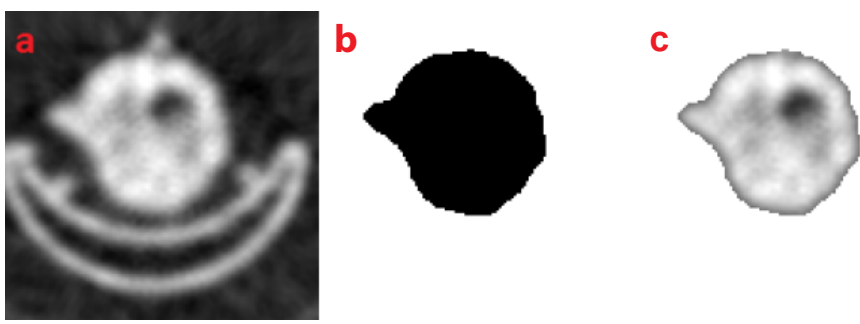

Figure 1: Manual editing for removal of the bed from the PET transmission image. Image (a) is the original PET transmission image. Image (b) is the binary mask used to extract the region of interest. Image (c) is the extracted region used in the automatic image registration programs. The masking was also done to the MR image to remove any background noise and the fiducial markers for automated image registration. mentioned above. The images are therefore edited to remove the bed from the image as shown in Figure 1. This is done by creating binary mask using ImageJ software, and editing out the bed from the image manually. The mask is then applied to the image, resulting in an image containing only the region of interest. For our Matlab GUI, no masking was required because the user registers the images based only on the location of the fiducial centroids in the MR image and original PET transmission image.

Prior to registration, the PET transmission and MR images were masked to remove the bed from the PET image. The fiducial markers were also removed from both images. These masked images were used for the automatic image registration programs AIR, FLIRT, and MIPAV. The parameters used to transform the masked PET transmission image to the MR image, were applied to the original PET transmission image containing the fiducial markers.

MAR-GUI: Our program, MAR-GUI, was written in Matlab and includes a simple interface created using Matlab's GUIDE application. The program allows the user to load a reference image and floating image in several file formats, in particular, Analyze 7.5 format. Figure 2 shows the MAR-GUI interface along with images registered using three fiducial markers. After loading the images, the user selects how many fiducial markers will be used for registration by selecting a number from a scroll box. The user then selects where the corresponding pairs of fiducials are located on both images using the "Place Fiducial" button; this is easily done since the program includes a "Find Centroid" button which will place markers on all of the centroids in the image. As the user selects the fiducial points, the coordinates are displayed in a table located under each image. After selecting the fiducial markers in both images, the user clicks the "Register" button and the resulting image is shown on the right side of Figure 2.

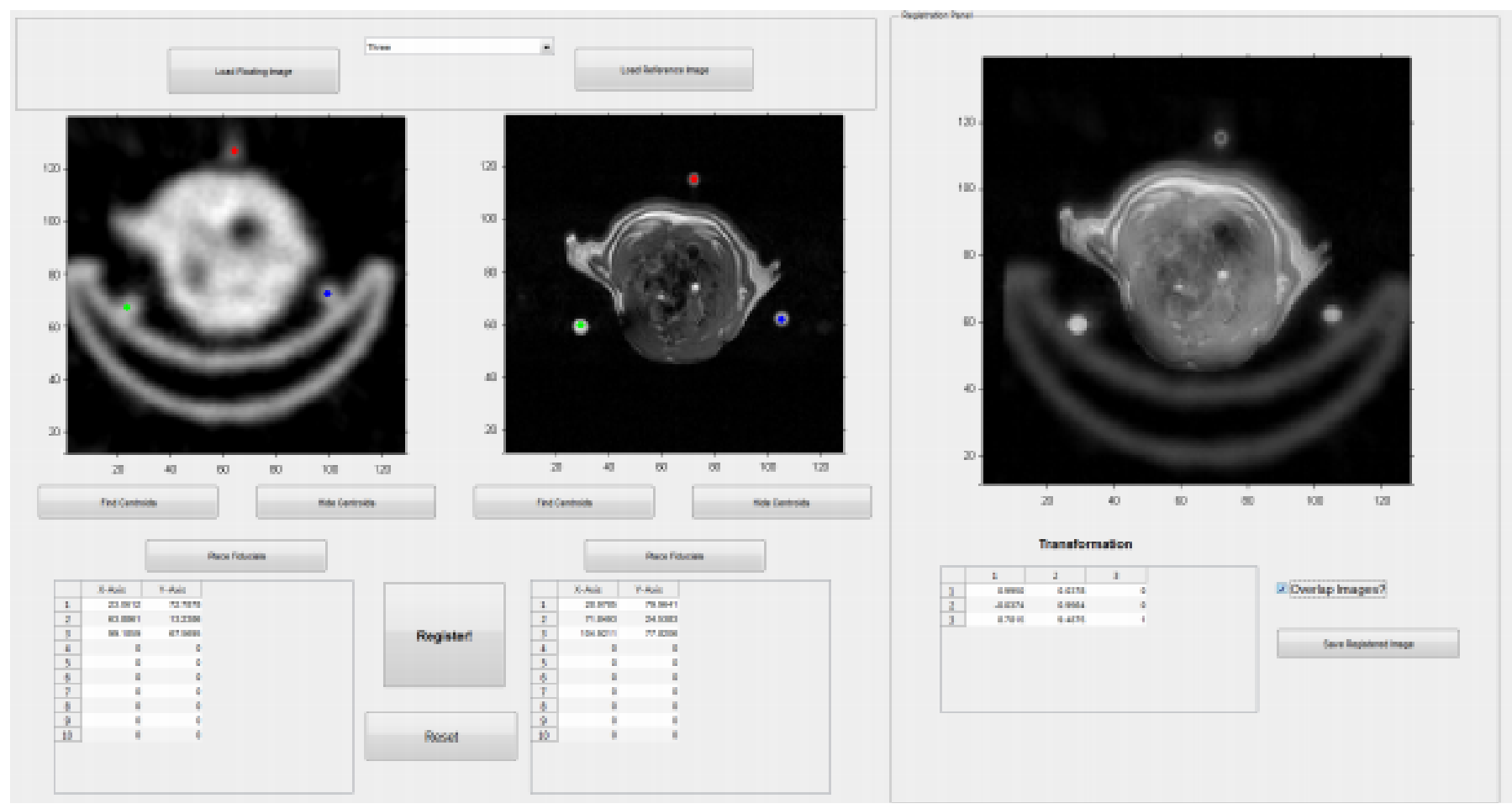

Figure 2: Screenshot of the Matlab GUI showing the registration of the floating PET transmission image with the corresponding MR reference image. The locations of the fiducials are displayed underneath the floating and reference images. The image at the far right has the transformed PET image overlaid on top of the MRI image. 
The transformation is calculated by minimization of the cost function in equation (2), and is then applied to the floating image and mapped onto the reference image. The transformation matrix is displayed under the transformed image, and has the form

$$
T=\left[\begin{array}{ccc}
A_{1,1} & A_{1,2} & 0 \\
A_{2,1} & A_{2,2} & 0 \\
T_{x} & T_{y} & 1
\end{array}\right]
$$

where $A$ is the affine matrix that includes the scale, skew, and rotation parameters. The terms $T_{x}$ and $T_{y}$ are the translation parameters along the $\mathrm{x}$ - and $\mathrm{y}$-axes, respectively. Note that equation (3) is essentially the right hand side of equation (1), with the translation vector $\boldsymbol{t}$ inserted into the $A$ matrix. A toggle button can be pressed to overlay the registered floating image onto the reference image, allowing for a visual check of the registration. If the registration was not sufficiently accurate, the user can click a "Reset" button to erase the current selected fiducial markers and select new fiducial markers.

Image registration assessment: The centroid of each fiducial marker was found using ImageJ by manually creating a region of interest (ROI) around each fiducial marker. The Euclidean norm of the fiducial marker centroids between the MR image and the transformed PET transmission image were calculated, yielding the fiducial registration error (FRE) in mm.

\section{Results and Discussion}

All of the registrations from the programs investigated in this study, i.e., FLIRT, AIR, and MIPAV were overlaid onto the MR image using ImageJ and are shown in Figure 3. It is clear that AIR was unable to register the images accurately; it appeared to have attempted to scale along the $y$-axis, but was not able to scale the $\mathrm{x}$-axis correctly. All other automatic registrations produced superior results compared to AIR, which is indicated in the visual registrations in Figure 3. MAR-GUI had the best results and was the only software that was able to visually overlap all three fiducials successfully.

The quantitative results are displayed in Table 2. The distance between the fiducial markers, or the FRE, is used as the registration accuracy metric. It can be seen that our Matlab program achieved the best registration, with an FRE Euclidean norm of $1.36 \mathrm{~mm}$. Most of the automated registration programs had an average FRE norm of 1.97 $\mathrm{mm}$, except for AIR, which had an FRE of $10.81 \mathrm{~mm}$. The automated image registration programs used the manually edited images as shown in Figure 1, while our Matlab program used the fiducial points of the unmasked images. The Matlab registration results are shown in Figure 3, which includes an overlap of the unregistered images and the registered images.

Fiducial registration, particularly MAR-GUI, has the potential to achieve better registration than automated registration algorithms, especially in difficult cases such as cardiac cell tracking studies. The registration accuracy was tested on an organ susceptible to motion (such as a beating heart) for the following reasons: it was the organ of interest in our present study, and image registration has been validated in other regions such as the brain.

The automatic image registration programs primarily use intensity based registration algorithms, which only work well when there is some commonality or relationship between the pixel intensities of the two images. This is due to the fact that the transformation is derived from the pixel values [16], as opposed to being derived from the
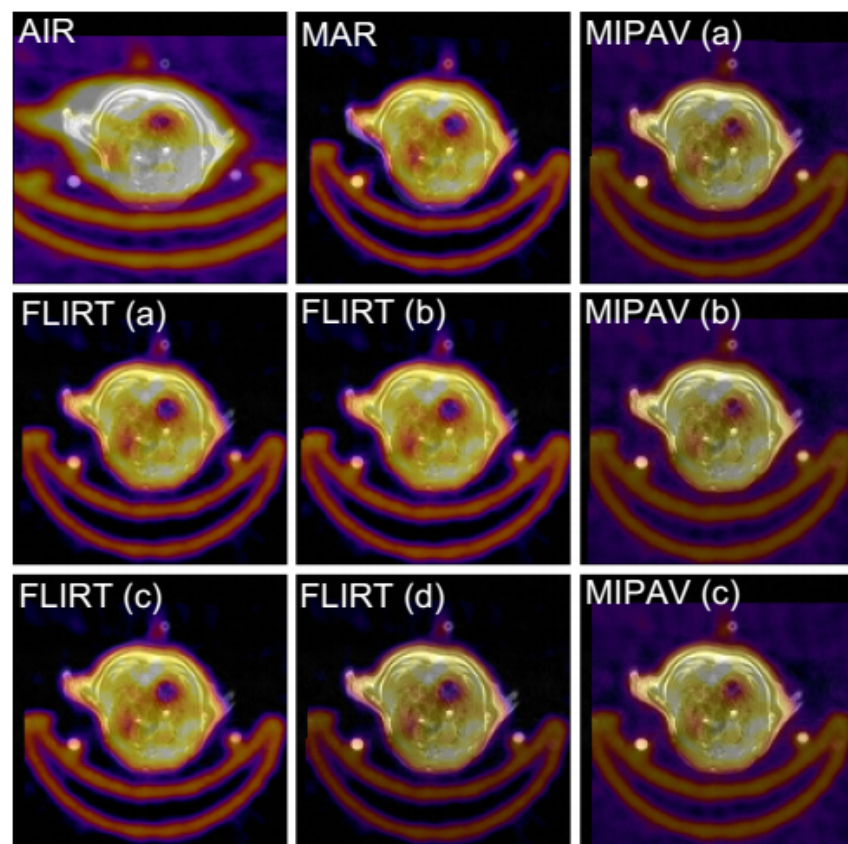

Figure 3: Visual comparison of the various registration algorithms. The cost functions for FLIRT include CR (a), NCC (b), MI (c), and NMI (d). The cost functions for MIPAV include CR (a), NCC (b), and NMI (c). The top middle image (MAR, short for MAR-GUI) was the only registration that was able to successfully overlap all three fiducial markers, whereas the other registrations were unable to overlap all of the fiducial markers, in particular, the top fiducial marker.

\begin{tabular}{|c|c|c|c|}
\hline Program-Method & Average X FRE & Average Y FRE & FRE Norm \\
\hline No Registration & 4.66 & 8.06 & 9.31 \\
\hline MIPAV - CR & 1.58 & 1.10 & 1.93 \\
\hline MIPAV - NCC & 1.43 & 1.05 & 1.77 \\
\hline MIPAV - NMI & 2.19 & 0.61 & 2.27 \\
\hline FLIRT - CR & 1.70 & 0.94 & 1.94 \\
\hline FLIRT - NCC & 1.11 & 0.93 & 1.45 \\
\hline FLIRT - MI & 1.69 & 1.09 & 2.01 \\
\hline FLIRT - NMI & 2.02 & 1.30 & 2.40 \\
\hline AIR - Woods & 10.75 & 1.10 & 10.81 \\
\hline MAR-GUI & 1.13 & 0.76 & 1.36 \\
\hline
\end{tabular}

Table 2: Fiducial registration error (FRE) results in units of $\mathrm{mm}$. The methods of registration are as follows: correlation ratio (CR), normalized cross correlation (NCC), mutual information (MI), normalized mutual information (NMI), and the Woods algorithm (Woods). The FRE is calculated for each fiducial and averaged for each axis. The final column is the Euclidean norm of the average $x$ and $y$ FRE.

geometrical landmark information. The PET emission data does not have a sufficiently strong commonality to the MR image to be directly registered. Since there is not enough structural information required for automatic registration, the PET transmission image must be used.

Landmark registration using fiducial markers is a simple registration method that has the ability to produce accurate results, but requires the object being scanned to remain fixed relative to the fiducial points. This can be very difficult to achieve in practice, with the exception of stereotactic devices [17]. Our study involved registration of external fiducial markers located around the specimen and assumes any motion, such as organ or body movement, to the rat was minimal. We discovered that injecting a high concentration of dual-labeled stem cells into localized regions can be used as temporary fiducial points. 
Citation: Dietz B, Alexander M, Elhami E, Xiang B, Deng J, et al. (2014) New Software for Multimodal Image Registration with Application in Cell Tracking Studies. J Stem Cell Res Ther 4: 239. doi:10.4172/2157-7633.1000239

Page 5 of 5
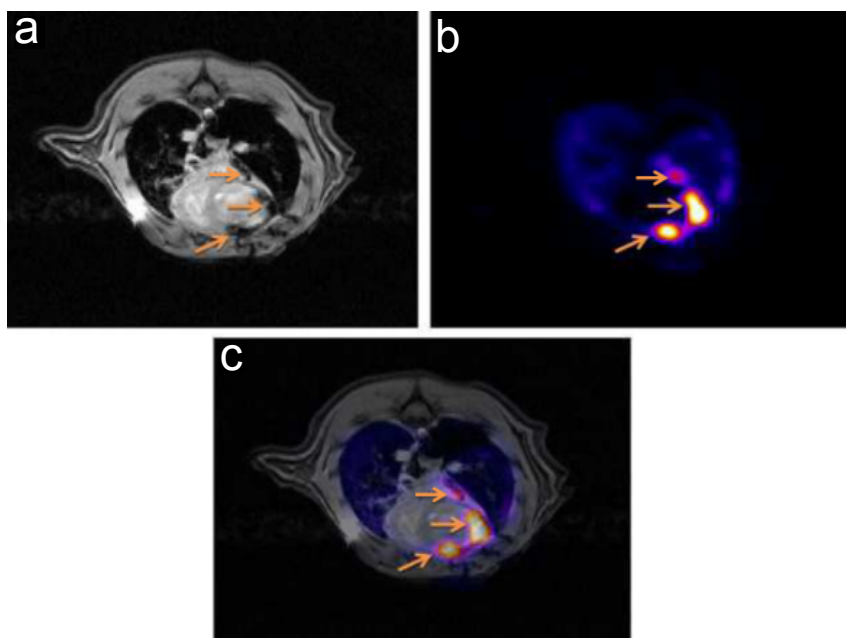

Figure 4: Registration through the use of fiducial points formed by injection of dual-labeled stem cells with PET-MR contrasts into heart tissue. Image (a) is the original MR image, image (b) is the original PET image, and image (c) is the transformed PET image overlaid on top of the MR image. The arrows point to the injection sites, which were used as fiducial markers for registration using MAR-GUI.

The temporary points located in the heart were used for registration as shown in Figure 4. This can be very useful as it avoids the tedious preparation of fiducial markers. However, further studies on the use of dual-labeled cells as internal fiducial markers need to be done to verify the accuracy of this method.

The Matlab program we developed requires user input, but the simplicity of the program renders training unnecessary. Our Matlab program does not require masking out any structures, since the registration is done based on the fiducial points chosen by the user. It should also be noted that it is successful specifically for regions, or data sets, which would not do well in automatic registration methods and contain either geometric landmarks or fiducial markers. The user can simply register the PET emission image directly to the MR image, without having to register to the PET transmission image first, as would be required for the automatic registration programs. Depending on the region being imaged landmark registration using various anatomical features or, as mentioned above, injection sites can be used for image registration instead of using fiducial markers.

The sensitivity of PET makes it ideal for stem cell tracking studies; however, without registration to an anatomical imaging modality such as MRI, the quantification of stem cells is difficult, because of the inaccurate delineation of the heart resulting in inaccurate \% injected doses (\%ID). The program MAR-GUI was used to achieve accurate quantitative data for PET-MRI stem cell tracking studies. The registration of the PET and MR images using MAR-GUI yielded definitive ROI's required to calculate the \%ID in the heart of rats injected with dual-labeled stem cells.

\section{Conclusion}

Registration of PET-MR images of an animal model injected with dual-labeled stem cells was simply and accurately registered using our Matlab developed software MAR-GUI. Other intensity based automatic image registration programs are able to register these images; however, they require an edited PET transmission image, which can be time consuming. Our method of registration is beneficial since PET transmission scans are not required, and the user only has to select the fiducial points in the PET emission and MR images for direct registration of the two. Our Matlab developed software is available free for download at www.sourceforge.net/p/matlabaffinereg.

\section{References}

1. Zhou R, Acton PD, Ferrari VA (2006) Imaging stem cells implanted in infarcted myocardium JACC 48: 2094-106. [PubMed]

2. Pauwels EK, Ribeiro MJ, Stoot JH, McCready VR, Bourguignon M, et al. (1998) FDG accumulation and tumor biology. Nucl Med Biol 25: 317-22. [PubMed]

3. Boellaard R (2009) Standards for PET image acquistion and quantitative data analysis. JNM 50: 11-20. [PubMed]

4. Chen IY, Wu JC (2013) Molecular imaging: the key to advancing cardiac stem cell therapy. Trends in Cardiovasc Med 23: 201-210. [PubMed]

5. Shen D, Liu D, Cao Z, Acton PD, Zhou R (2008) Coregistration of magnetic resonance and single photon emission computed tomography images for noninvasive localization of stem cells grafted in the infarcted rat myocardium. Mol Imaging Biol 1: 24-31. [PubMed]

6. Qiao R, Yang C, Gao M (2009) Superparametric iron oxide nanoparticles: from preparations to in-vivo MRI applications. J Mater Chem 19: 6274-93. [PubMed]

7. Zitova B, Flusser J (2003) Image registration methods: a survey. Image and Vision Computing 21: 977-1000. [PubMed]

8. Jenkinson M, Bannister P, Brady JM, Smith SM (2002) Improved optimization for the robust and accurate linear registration and motion correction of brain images. Neuroimage 2: 825-41. [PubMed]

9. Woods RP, Mazziotta JC, Cherry SR (1993) MRI-PET registration with automated algorithm. Journal of Computer Assisted Tomography 17: 536-46. [PubMed]

10. McAuliffe MJ, Lalonde FM, McGarry D, Gandler W, Csaky K, et al. (2001) Medical image processing, analysis and visualization in clinical research. IEEE CBMS 381-86. [PubMed]

11. Fitzpatrick JM, West JB (2001) The distribution of target registration error in rigid-body point-based registration. IEEE Transactions on Medical Imaging 20: 917-27. [PubMed]

12. Elhami E, Dietz B, Xiang B, Deng J, Wang F, et al. (2013) Assessment of three techniques for delivering stem cells to the heart using PET-MR imaging. Eur $\mathrm{J}$ Nucl Med Mol Imaging 3: 72. [PubMed]

13. Elhami E, Goertzen AL, Xiang B, Deng J, Stillwell C, et al. (2011) Viability and proliferation potential of adipose-derived stem cells following labeling with a positron-emitting isotope. Eur J Nucl Med Mol Imaging 38: 1323-34. [PubMed]

14. Cherry SR, Sorenson JA, Phelps ME (2003) PA: Saunders.

15. Press WH, Teukolsky SA, Vetterling WT, Flannery BP (2007) Numerica Recipes. The Art of Scientific Computing, Cambridge, UK: Cambridge.

16. Hill DL, Batchelor PG, Holden M, Hawkes DJ (2001) Medical image registration. PMB 46: R1-R45. [PubMed]

17. West JB, Fitzpatrick JM, Toms SA, Maurer CR, Maciunas RJ (2001) Fiducial point placement and accuracy of point-based rigid body registration. Neurosurgery 48: 810-17. [PubMed] 